\title{
Effect of Grip Training through Proto-type Silicone Dynamic Wrist Devices on Grip Strength and Wrist Flexor Tension in Chronic Stroke Patients
}

\author{
Daehwan Lee ${ }^{1}$, Jungho Lee ${ }^{2}$ \\ ${ }^{1}$ Professor, Dept. Physical Therapy, Andong Science College, Republic of Korea, \\ dhlee8510@naver.com \\ ${ }^{2}$ Professor, Dept. Physical Therapy, Kyungdong University, Republic of Korea, ljhcivapt@naver.com
}

Corresponding author: Jungho Lee

\begin{abstract}
The hand functions have very important roles in the patient's daily life, and if the hand strength is weak or muscle tension is abnormal, the patient has a major disability in moving the hand. If the tension of the hand muscle is too low, the patient cannot contract the muscle, and if the tension of the hand muscle is too high, the contraction of the muscle cannot be controlled by itself, and the movement of the joint cannot be made. Muscle tension in stroke patients increases over time and stiffness occurs, which eliminates joint movement. This study attempted to change muscle tension and increase muscle strength by performing hand grip training with a silicone dynamic wrist training mechanism. The subjects of this study were a total of 20 patients 6 months after stroke, and the effectiveness of a new hand training device was confirmed by measuring hand strength and modified ashworth scale (MAS). The general characteristics of the study subjects are that the average age was $60.32 \pm 4.72$ years, the average height was $168.53 \pm 8.65 \mathrm{~cm}$, and the average weight was $65.33 \pm 11.23 \mathrm{~kg}$. The average MMSE-K score was $27.23 \pm 1.01$. The increase in hand strength in chronic stroke patients was $2.80 \pm 1.52$, which was statistically significant. In addition, the muscle tension changed from $2.60 \pm 0.65$ to $2.33 \pm 0.59$ and was statistically significant. The newly developed hand trainer is thought to have a positive effect on the improvement of hand function in patients with stroke.
\end{abstract}

Keywords: Hand Power, Ashworth Scale, Exercise Device, Rehabilitation

\section{Introduction}

Stroke is caused by ischemic infarction in the brain and cerebral hemorrhage. Stroke patients have difficulty living independently due to severe motor disorders caused by chronic disorders such as motor function, cognition, perception, and sensation. In addition, most stroke patients have problems with upper limb function, and patients with severe upper limb muscle damage have a very high risk of spasticity in their arms. In addition, as stroke progresses to the chronic stage, the patient's hands and wrists become bent. This contracture generally occurs between six and eight weeks after stroke. The contracture and paralysis of the upper extremities place significant restrictions on daily life, affecting washing, dressing, eating, and wheelchair use. If not paying proper attention to various activities, it is likely to cause secondary damage to the patient's fingers, joints, and surrounding soft tissue, and if it is not prevented by rehabilitation, fist-shaped deformation occurs[1].

Spasticity and contracture are the most common complications that cause exercise restrictions after

Received: July 25, 2021; $1^{\text {st }}$ Review Result: September 13, 2021; $2^{\text {nd }}$ Review Result: October 26, 2021 Accepted: November 30, 2021 
stroke, and stretching plays a very important role in preventing these complications. To prevent muscle contracture, muscles have to be stretched for at least six hours a day. Orthopedic surgeons recommend that a splint should be worn for a long period to prevent muscle contracture. However, several studies have shown limited evidence of the effectiveness of splints, and clinically, many stroke patients cannot withstand static stretching for more than six hours a day. It has been reported that about $50 \%$ of stroke patients cannot perform static stretching for more than six hours due to discomfort[2]. In addition, stretching with the use of a static device for a long time can cause spasm in the wrist and fingers to increase due to pain stimulation in the hand and lower arm, and continuous wearing of the orthosis can result in pain and bedsores. It is assumed that this pain and discomfort is caused by fluctuations in the static orthosis on effected side and spasticity on the other. At the moment of severe spasticity, the wrist tightens, which increases pain, pressure, and muscle tone due to sensory pain stimulation. At other moments, your wrist may relax and lack stretching. If static orthopedic surgery is not well tolerated, contraction may occur, and a more active intervention, such as botulinum toxin treatment and surgery, may be required to prevent fist clumping[3].

Continuous wearing of static aids can cause various disorders to the patient's muscles, especially spasticity due to increased muscle tension, and muscle elasticity can be lost. In addition, the contracture of joints and ligaments may result in loss of mobility of joints putting static aids on, and the problem of constructing joints and ligaments further hinders the action of muscles. In particular, since spasticity of the hand is destructive, improvement of the shoulder or elbow may not be appropriate to make certain overall improvement of the upper limb[4].

In previous studies, various outcome measurements for upper limb function were used and training was conducted, but few studies have focused on hand movement. Some studies on improvement of hand function describe injecting drugs such as BTX-A into muscles or electrical stimulation, but studies confirming the relaxation of hand flexion muscles through training are insufficient[3].

About $60-70 \%$ of stroke patients experience upper limb dysfunction, and $20 \%$ of stroke survivors are dependent on the help of others in their daily lives. This disability of upper limb function is considered one of the biggest obstacles in the daily lives of stroke patients and should be studied by many researchers now and in the future.

The upper limb consists of the upper arm, the lower arm, and the hand. The hand, in particular, performs functional activities through delicate movements. Hands are used in all aspects of a patient's daily life, such as eating, washing of the face, showering, dressing, cooking, and cleaning. The grip function of the hand is especially important for performing these actions. It consists of a strong grip, a precision grip, and a hook-type grip. All grips other than hook-type grip are very important in the action of the thumb. In the case of fixtures, it is used for movements that require delicacy such as keys, needles, pencils, and chopsticks through the thumb and index fingers[5].

Sufficient muscle strength is essential to perform such functional operations, and muscle tension capable of contracting muscles is important. If muscle strength is insufficient, the patient will not be able to hold an object or maintain movement. If muscle tension is too low, the patient will not be able to produce muscle contraction, and if the muscle tension is too high, this will prevent joint movement and the patient will not be able to perform functional movements. Therefore, securing muscle tension and muscle strengthening training are among the most important factors that can create functional movements in stroke patients.

Previous studies with elderly patients have shown that the power to hold is an indicator of an individual's health status. One study reported that a decline in grip strength in female patients was associated with a decrease in bone density. Grip strength has been found to be a useful indicator for predicting early death, early disability development, postoperative complications, post-fracture hospitalization duration, and cognitive decline in the elderly. A study of the relationship between grasping power and perception evaluated cognitive function in 76 elderly people using the clinical 
dementia rating, the mini mental state test - Korean version (MMSE-K), the Roton scale, and evaluation of daily life activity[6].

Since the reliability suitable for the grip strength has been shown in stroke patients and the patients with cognition disease, it has been reported that assessment based on grip strength can be applied not only to baseline dementia patients but also to patients with minimal and severe dementia. A study of older Mexican Americans showed that people with weak grip power tend to score low in the MMSE over time.

Neuroscientifically, repeatedly and task-oriented training of peripheral movements promotes neuroplasticity in the brain, which, in turn, can increase the performance of peripheral movements. According to this theory, physical therapy methods can be effectively used for stroke patients[7]. In addition, muscle strength can only be improved through resistance exercises. Manual or active motion can maintain the movement of the joints and secure the elasticity of soft tissue, but it cannot improve muscle strength. Therefore, it is recommended that patients use assistants for training or short-time task performance, as the use of assistants can reduce the performance of the patient's muscle strength in the long run. In this study, in order to suppress spasticity of the patient's hand and finger flexors and maintain muscle elasticity, the effect of a silicone hand trainer on grip and muscle tension in stroke patients was investigated[8].

The use of upper limb aids has a long history, and many developments in this field have been made possible through military funds. Many casualties occurred through major wars such as the World War I and World War II, and several aids are being developed to assist them in their lives. Patients using traditional physical therapy or aids generally suffer from loss of upper limb function, dependence on caregivers, and poor quality of life. For this reason, it is necessary to develop an adjuvant device to enhance the quality of life of patients, and research into the development of such devices is currently being actively conducted. In the United States, the development of products that assist muscle movement through electrical signals or artificial intelligence using a combination of electromyography and aids is actively underway. However, these mechanical and artificial intelligence devices are difficult to use in patients at present due to cost problems[9]. In addition to developing solutions for the near future, it is necessary to forward the quality of life through aids that can be used by patients today.

Therefore, the researchers propose a new type of silicone hand trainer that is easy to attach and detach and allows patients to perform hand strength training easily. In the past, hand aids were used for simple stretching or fixing and included springs, which made them very uncomfortable to wear and detach. They were also complicated in structure, making them difficult to use and subject to frequent failures. Therefore, we propose a new type of silicone hand trainer that is easy to attach and detach and allows patients to perform hand strength training easily.

Hand function impairment in stroke patients can be evaluated by Brunnstrom recovery stage, maximum grip, the Fugl-Meyer assessment, the modified Ashworth scale (MAS), and the effect of repeated training on stroke patients' maximum grip and improvement of future silicone hand trainers.

In this study, it was investigated whether it was possible to change the tension of the flexor muscles of the hand through a silicone hand trainer and confirm the increase in muscle strength through MAS and hand power.

\section{Methods}

\subsection{Subjects}

For this experiment, outpatients from D Hospital in D City, Korea were recruited. The inclusion criteria were (1) patients who had developed a stroke for more than 6 months, (2) upper limb 3-5, (3) hemiplegic patients without adjuvant devices classified as Brunnstrom's motor recovery stage, (4) ability 
to understand and follow oral instructions, (5) ability to walk more than $10 \mathrm{~m}$ without adjuvant. (6) Patients without orthopedic disease in the upper extremity and (7) Mini-Mental State Examination, Korean version (MMSE-K) score of 24 or higher. The exclusion criteria were (1) visual, auditory, or vestibular disorders, and (2) functional problems of the upper limb due to other neurological problems unrelated to stroke.

According to the inclusion and exclusion criteria, a total of 20 patients, including 15 men and 5 women, were recruited. The average age was $60.32 \pm 4.72$ years, the average height was $168.53 \pm 8.65$ $\mathrm{cm}$, and the average weight was $65.33 \pm 11.23 \mathrm{~kg}$. The average MMSE-K score was $27.23 \pm 1.01$. Cerebral infarction was a seizure type of all patients, and the average time elapsed after stroke was 13.23 \pm 3.13 months.

[Table 1] General Characters

\begin{tabular}{|c|c|c|}
\hline & Mean & SD \\
\hline Age & 60.32 & 4.72 \\
\hline Height & 168.53 & 8.65 \\
\hline Weight & 65.33 & 11.23 \\
\hline MMSE-K & 27.23 & 1.01 \\
\hline Duration after stroke & 13.23 & 3.13 \\
\hline
\end{tabular}

The study was conducted according to the ethical standards described in the Declaration of Helsinki. All participants submitted prior written consent prior to the study. For comparative analysis, patients compared hand grip strength and muscle tension before and after training to wear assistants.

\subsection{Flow Chart}

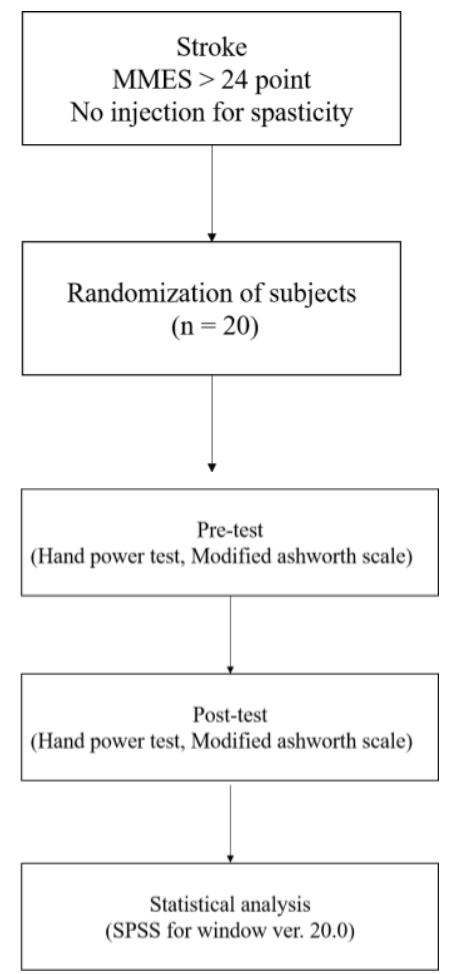

[Fig. 1] Flow Chart 


\subsection{Study Hypothesis}

If the muscle tension is abnormal, it causes a decrease in muscle strength. In this study, it was assumed that silicone tension could improve the muscle strength of the hand by relieving muscle tension in stroke patients.

H0 : Silicon tension cannot change muscle tension.

$\mathrm{H} 1$ : The tension of silicone can change the tension of the muscles, thereby efficiently increasing the muscle strength.

\subsection{Hand Power}

In stroke patients, it take on that the muscle tension increased as they progressed chronic, resulting in a decrease in muscle strength. It is assumed that muscle strength will also improve if the muscle tension approaches normal. This test was evaluated through a grip power as an evaluation to measure the hand strength of stroke patients. Each patient was instructed to perform the test three times and the average of the three measurements was calculated. The grip tester used in this study is PGF-1000 from pro-specs, with a maximum measurement of $90 \mathrm{Kg}$ and a unit of $100 \mathrm{~g}$.

\subsection{Modified Ashworth Sacle (MAS)}

As an evaluation of muscle tension, the tension of the patient's hand muscles was evaluated using MAS, which is commonly used internationally. Spasticity was defined by Jim Lance in 1980 as a component of upper motor neuron syndrome and as an increase dependent on the rate of muscle stretch reflection associated with muscle tone increase. Spasticity have a wide range of etiology, including brain damage, stroke, cerebral palsy, multiple sclerosis, trauma, and spinal cord damage[9].

Spasticity, defined as a response dependent on the speed of the muscle to manual stretching, is one of the most commonly recognized sequences of central nervous system disorders. While the role actually played by convulsions in motor dysfunction may be less than suggested by some researchers, reactive muscle contracture may limit the force demonstrated by agonist muscles for spontaneous movement[10].

[Table 2] Modified Ashworth Scale (MAS)

\begin{tabular}{|c|l|}
\hline Grade & The grades of MAS are as follows: \\
\hline 0 & Muscle tone does not increase. \\
\hline 1 & $\begin{array}{l}\text { When a slight increase in muscle tone, the affected part moves in flexion or extension, it is held } \\
\text { and released at the end of the range of motion (ROM) or appears as minimal resistance. }\end{array}$ \\
\hline $1+$ & A slight increase in muscle tone with minimal resistance in the rest of the ROM (less than half). \\
\hline 2 & $\begin{array}{l}\text { Most ROMs showed a more significant increase in muscle tone, but the affected part moved } \\
\text { easily. }\end{array}$ \\
\hline 3 & Significant increase in muscle tone and passive movement are difficult. \\
\hline 4 & Rigid while almost flexion or extension of affected parts. \\
\hline
\end{tabular}

\subsection{Data Analysis}

Data analysis was performed using the average of the three measurements for each test. The data were expressed as mean \pm standard deviations. The paired t-test was used to compare the measurements performed before and during the patient's exercise with the new hand divice. Statistical analysis was performed using IBM SPSS Statistics 20.0 for Windows. 


\section{Results}

\subsection{Hand Power}

This test was evaluated through a grip as an evaluation to measure the hand strength of stroke patients. Each patient was instructed to perform the test three times and the average of the three measurements was calculated. The mean power to complete the hand power was $15.32 \pm 3.58 \mathrm{~kg}$ before and $18.12 \pm$ $5.10 \mathrm{~kg}$ after exercise. The difference was statistically significant.

[Table 2] Grip Power (kg)

\begin{tabular}{|c|c|c|c|}
\hline Group & Mean & $\mathrm{SD}$ & $p$ \\
\hline Pre & 15.32 & 3.58 & \multirow{2}{*}{$0.03 *$} \\
\hline Post & 18.12 & 5.10 & \\
\hline \multicolumn{4}{|c|}{$\begin{array}{l}\text { *SD: standard deviation } \\
* p<0.05 *,<0.01 * *,<0.001 * * * \\
* \text { unit: seconds }\end{array}$} \\
\hline \multicolumn{4}{|l|}{25} \\
\hline \multicolumn{4}{|l|}{20} \\
\hline \multicolumn{4}{|l|}{15} \\
\hline \multicolumn{3}{|l|}{10} & \\
\hline
\end{tabular}

[Fig. 2] Grip Power

\subsection{Modified Ashworth Sacle (MAS)}

The mean score of MAS was $2.60 \pm 0.65$ before and $2.33 \pm 0.59$ after exercise with wrist brace. The difference was statistically significant.

[Table 3] Modified Ashworth Scale

\begin{tabular}{|c|c|c|c|}
\hline Group & Mean & SD & $p$ \\
\hline Pre & 2.60 & 0.65 & \multirow{2}{*}{$0.000 * * *$} \\
\hline Post & 2.33 & 0.59 & \\
\hline
\end{tabular}

*SD: standard deviation

$* p<0.05 *,<0.01 * *,<0.001 * * *$

*unit: $\mathrm{mm}\left(10^{-3} \mathrm{~m}\right)$

\section{Discussion}

Stroke patients suffer from muscle weakness, cognitive dissonance, and self-acceptance sensory disorders throughout the body, causing balance, adjustment, and functional motor disorders. This study 
investigates the effect of hand bending training on hand muscle strength and hand bending muscle tension using a newly manufactured silicone hand assistant.

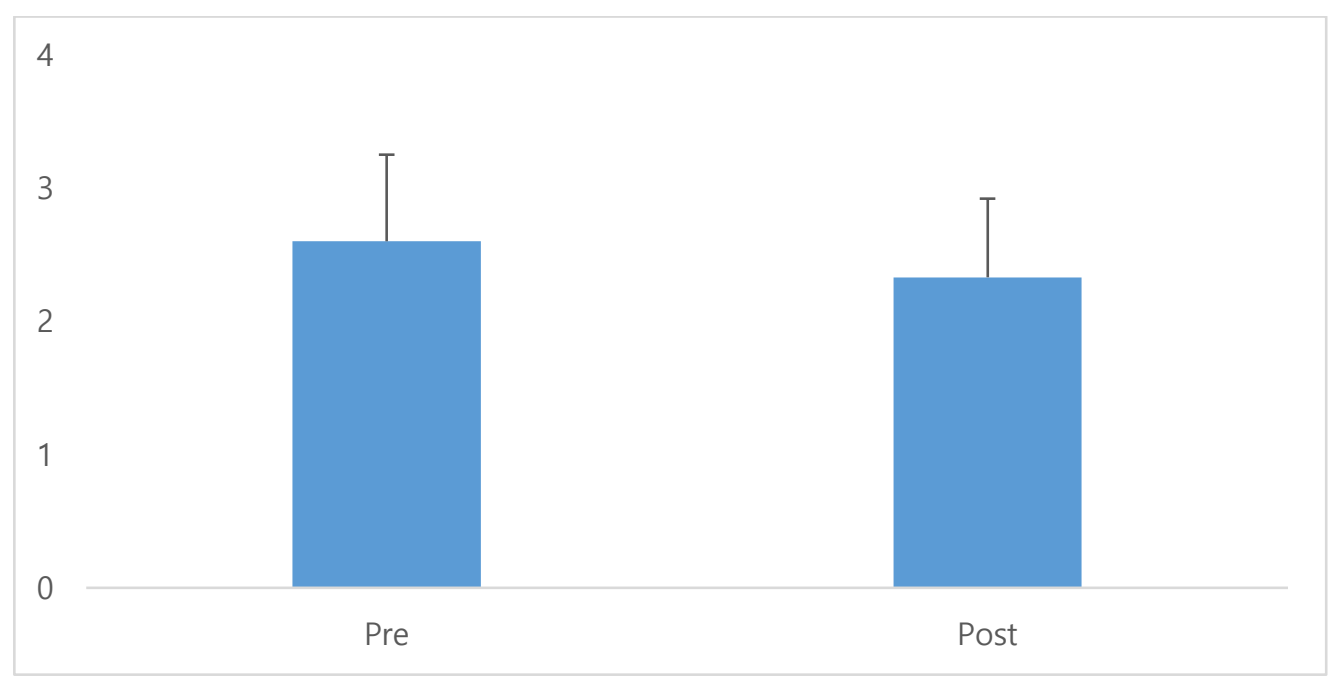

[Fig. 3] Modified Ashworth Scale

The elastic nature of silicone makes it suitable for stretching and resistance training. Currently, silicone is used to manufacture rehabilitation training tools such as TheraBand and gym balls and has the advantage of being able to apply various resistance forces to users depending on the degree of elasticity. It is extremely durable, and its simple structure makes it easy to use. Since silicone can also apply structural pressure, it can apply pressure to the fingertips to provide sensory stimulation[11].

To confirm the basic effect of the prototype product, simple repetitive training was performed on the patient's hand, and the resulting increase in muscle strength was confirmed. Since the training was performed for only a short period, the increase in muscle strength was not significant enough to affect the patient's daily life. However, it is expected that long-term training would have a significant effect on a patient's daily life.

The currently used training machine was mass produced using a silicone manufacturing mold device. This may have reduced the effectiveness of the training because it did not reflect individual patient differences. However, it should be possible to vary the size in the future to provide customized training for patients[4]. In addition, since all the elasticity of silicone was uniformly applied, it is expected that the effect of training according to individual basic abilities was different.

In the case of muscles, active exercise should be performed to maintain muscle elasticity, while manual exercise should be performed to maintain joint mobility, and resistance exercise can lead to changes in muscle tension and muscle strength. Therefore, the device should be worn for a short time, while the remaining muscle strength should be improved as much as possible through resistance training because hard and static aids cause joint contracture and decrease muscle elasticity[12]. Muscles are dominated by nerves, but restructuring damaged areas of nerves is possible through muscle movement[6].

Previous studies have reported that dynamic aids resulted in less pain for patients than static aids and that wrist spasms increased in patients who wore static aids for a long time. This can be seen as having an adverse effect on the patient, as spasms increase muscle tension[13][14].

So far, existing studies have not focused on the use of dynamic orthopedics when preventing and treating wrist contractions after stroke. Both studies of McPherson and Scherling showed a decrease in hypersensitivity through dynamic splints in hemiplegic arms of chronic stroke patients, but did not study the effect on contraction[15][16]. Despite the lack of research, more dynamic orthopedics have been 
developed in recent years.

Two recent systematic reviews demonstrated that static orthopedic surgery does not affect contraction prevention or spasticity reduction[17][18]. Unlike these studies, the researchers found a significant effect of dynamic splint on the muscle length of the wrist. For this reason, it is possible that the objective improvement of wrist contraction reduction observed in this study is mainly due to new orthosis based on low load and long-term stretching techniques. Static rigid orthopedic surgery can cause pain by increasing stress on the fingers and wrist joints due to fluctuations in the level of spasticity[19,][20].

This study confirms that there is no dynamic orthopedic effect on spasticity measured by MAS reported in previous studies[19][21]. Six months after orthopedic surgery, there was no significant change in spasticity, but there was a difference in spasticity treatment in spasticity treatment. One of the patients discontinued botulinum toxin injection for three months, and the other reduced the dosage and frequency after botulinum toxin injection. This may indicate that dynamic orthosis affects spasticity to some extent, but further research is needed[3][21].

There was only one patient who stopped putting it on after four months, even though all of the included patients could not tolerate static orthosis. However, the reason was irrelevant to the acceptability of splint. This orthosis did not fulfil the expectations of patients who expected to improve by lowering the degree of spasticity in their forearms instead of maintaining the length of the wrist and finger flexors[19]. Due to a lack of improvement, the patient decided to stop orthosis treatment. It is important to explain the effects that the patient can expect in the future and to monitor whether the possible effects meet the patient's expectations.

There was only one patient who stopped wearing it after four months, even though all of the included patients could not tolerate static splint. However, the reason was irrelevant to the acceptability of splint. This splint did not fulfil the expectations of patients who expected to improve by lowering the degree of convulsions in their forearms instead of maintaining the length of the wrist and finger flexors. Due to a shortage of enhancement, the patient decided to stop orthopedic treatment. It is important to explain the effects that the patient can expect in the future and to monitor whether the possible effects meet the patient's expectations.

\section{Conclusion}

This study has some limitations, which must be considered. The sample size was small $(\mathrm{n}=20)$. Therefore, the results should be confirmed in a large study. However, even this small group was sufficient to show a significant effect on the length of the wrist and finger bending muscles. It should be noted that this study is the first to investigate the durability and effectiveness of newly developed dynamic splint. In addition, since comparisons were made between outpatients, changes in the patients' living environment could not be considered. Future studies that control for specific variables could produce stronger results. While the results propose that the employ of a dynamic orthopedic device is promising for stroke rehabilitation, more researches are needed on the effectiveness of dynamic orthopedic devices.

New forms of silicone wrist and finger exercise will allow chronic stroke patients' finger and wrist bending exercises to change the tension of the bent muscles of the wrist, increase muscle strength, and have greater effects by applying new exercise programs in the future.

\section{References}

[1] S. A. Aukje, G. L. Ingrid, G. M. Jan-Willem, Tolerance and effectiveness of a new dynamic hand-wrist orthosis in chronic stroke patients, NeuroRehabilitaion, (2013), Vol.33, No.2, pp.225-231, DOI: 10.3233/NRE-130949 
[2] D. Stefanie, D. D. Brianna, P. Jessica, T. Daniel, N. Jonathan, Case Report on the Use of a Custom Myoelectric ElbowWrist-Hand Orthosis for the Remediation of Upper Extremity Paresis and Loss of Function in Chronic Stroke, Military medicine, (2017), Vol.182, No.7, pp.e1963-e1968, DOI: 10.7205/MILMED-D-16-00399

[3] J. M. Lee, J. M. Grecies, S. B. Park, K. H. Lee, J. Y. Lee, J. H. Shin, Botulinum toxin injections and electrical stimulation for spastic paresis improve active hand function following stroke, Toxins, (2018), Vol.10, No.11, p.426, DOI: $10.3390 /$ toxins 10110426

[4] H. Abe, A. Michimata, K. Sugawara, N. Sugaya, S.-I. Izumi, Improving gait stability in stroke hemiplegic patients with a plastic ankle-foot orthosis, The Tohoku Jounal of Experimental Medicine, (2009), Vol.218, No.3, pp.193-199, DOI: $10.1620 /$ tjem.218.193

[5] B. J. Darter, J. M. Wiken, Gait training with virtual reality-based real-time feedback: improving gait performance following transfemoral amputation, Physical Therapy, (2011), Vol.91, No.9, 1385-1394, DOI: 10.2522/ptj.20100360

[6] Y. H. Park, Y. M. Kim, B. H. Lee, An ankle proprioceptive control program improves balance, gait ability of chronic stroke patients, Journal of Physical Therapy Science, (2013), Vol.25, No.10, pp.1321-1324, DOI: 10.1589/jpts.25.1321

[7] L. A. B. Ferreira, H. P. Neto, L. A. C. Grecco, T. C. L. Christovão, N. A. Duarte, R. D. Lazzari, M. Galli, C. S. Oliveira, Effect of Ankle-foot Orthosis on Gait Velocity and Cadence of Stroke Patients: A Systematic Review, Journal of Physical Therapy Science, (2013), Vol.25, No.11, pp.1503-1508, DOI: 10.1589/jpts.25.1503

[8] D. C. Kerrigan, M. E. Karvosky, P. O. Riley, Spastic paretic stiff-legged gait: joint kinetics, American Journal of Physical Medicine \& Rehabilitation, (2001), Vol.80, No.4, pp.244-249, DOI: 10.1097/00002060-200104000-00002

[9] H. Andrew, K. Stephen, Modified Ashworth Scale, StatPearls Publishing, (2021)

[10] R. W. Bohannon, M. B. Smith, Interrater Reliability of a Modified Ashworth Scale of Muscle Spasticity, Physical Therapy, (1987), Vol.67, No.2, pp.206-207, DOI: 10.1093/ptj/67.2.206

[11] F. T . Sarah, M. K. Ruth, Effects of an Ankle-Foot Orthosis on Balance and Walking After Stroke: A Systematic Review and Pooled Meta-Analysis, Archives of Physical Medicine and Rehabilitation, (2013), Vol.94, No.7, pp.1377-1385, DOI: 10.1016/j.apmr.2012.12.025

[12] C. D. M. Simons, E. H. van Asseldonk, H. van der Kooij, A. C. H. Geurts, J. H. Buurke, Ankle-foot orthoses in stroke: effects on functional balance, weight-bearing asymmetry and the contribution of each lower limb to balance control, Clin Biomech (Bristol, Avon), (2009), Vol.24, No.9, pp.769-775, DOI: 10.1016/j.clinbiomech.2009.07.006

[13] J. Lewallen, J. Miedaner, S. Amyx, J. Sherman, Effect of three styles of custom ankle foot orthoses on the gait of stroke patients while walking on level and inclined surfaces, Jounal of Prosthetics and Orthotics, (2010), Vol.22, No.2, pp.78-83, DOI: 10.1097/JPO.0b013e3181d84767

[14] H. J. Hillstrom, J. Song, A. P. Kraszewski, J. F. Hafer, R. Mootanah, A. B. Dufour, B. S. Chow, J. T. Deland, Foot type biomechanics part 1: structure and function of the asymptomatic foot, Gait Posture, (2013), Vol.37, No.3, pp.445-451, DOI: 10.1016/j.gaitpost.2012.09.007

[15] J. J. McPherson, A. H. Becker, N. Franszczak, Dynamic splint to reduce the passive component of hypertonicity, Archives of Physical Medicine and Rehabilitation, (1985), Vol.66, No.4, pp.249-252, DOI: 10.1016/00039993(85)90162-5

[16] E. Scherling, H. Johnson, A tone-reducing wrist-hand orthosis, American Journal of Occupational Therapy, (1989), Vol.43, No.9, pp.609-611, DOI: 10.5014/ajot.43.9.609

[17] K. H. Kim, S. H. Jang, A Convergence study on effects of progressive proprioceptive motor program training on proprioception and balance ability in chronic stroke patients, Journal of the Korea Convergence Society, (2018), Vol.9, No.10, pp.81-91, DOI: 10.15207/JKCS.2018.9.10.081

[18] J. H. Carr, R. B. Shepherd, L. Nordholm, D. Lynne, Investigation of a new motor assessment scale for stroke patients, Physical Therapy, (1985), Vol.65, No.2, pp.175-180, DOI: 10.1093/ptj/65.2.175

[19] N. A. Lannin, L. Ada, Neurorehabilitation splinting: Theory and principles of clinical use, NeuroRehabilitation, (2011), Vol.28, No.1, pp.21-28, DOI: 10.3233/NRE-2011-0628 
Effect of Grip Training through Proto-type Silicone Dynamic Wrist Devices on Grip Strength and Wrist Flexor Tension in Chronic Stroke Patients

[20] S. F. Tyson, R. M. Kent, The effect of upper limb orthotics after stroke: A systematic review, NeuroRehabilitation, (2011), Vol.28, No.1, pp.29-36, DOI: 10.3233/NRE-2011-0629

[21] O. M. Katalinic, L. A. Harvey, R. D. Herbert, A. M. Moseley, N. A. Lannin, K. Schurr, Stretch for the treatment and prevention of contractures, Cochrane Database of Systematic Reviews, (2010), Vol.8, No.9, pp.CD007455, DOI: 10.1002/14651858.CD007455.pub2 